\title{
Does quality of care entail environmental impact? A blind spot in our knowledge
}

\author{
Leonie M. S. Veltman ${ }^{1}$, Diana M. J. Delnoij ${ }^{1,2}$, Hans C. Ossebaard ${ }^{* 1,3}$ \\ ${ }^{1}$ National Health Care Institute, Diemen, The Netherlands \\ ${ }^{2}$ Erasmus School of Health Policy \& Management, Erasmus University Rotterdam, The Netherlands \\ ${ }^{3}$ Department of Medical informatics, Amsterdam University Medical Centers, The Netherlands
}

Received: May 3, 2020

DOI: $10.5430 /$ ijh.v6n2p74
Accepted: July 26, 2020

URL: https://doi.org/10.5430/ijh.v6n2p74
Online Published: August 13, 2020

\begin{abstract}
This scoping review examines the conceptual relationship between the terms "environmental sustainability" and "quality of care" as used in academic studies on health care. We performed searches in Scopus and PubMed looking for potential semantic and practical associations between sustainability and quality of care, including potential conflicts. For the first part about associations, 11 search strings were used resulting in 1,488 studies of which 8 were eventually selected for analysis. For the latter part about conflicts, 4 search strings were used resulting in 45 studies of which 6 remained for analysis. Information about the following aspects was extracted from the studies that were included: interpretation and definition of sustainability, dimensions of quality of care, and tensions between quality and sustainability. Merely a few studies address a relationship between environmental sustainability and quality of care. Only "patient-centredness" and "safety" are associated with sustainability in academic literature. "Effectiveness" is rather interpreted as opposing it. "Efficiency" seems to be both associated and opposed to sustainability. The conceptual relationship between environmental sustainability and quality of care has not been thouroughly examined in academic studies which implies a blind spot in our knowledge. Only one study reports on conceptual and practical work for incorporating sustainability as a dimension of health care quality.
\end{abstract}

Key Words: Quality of care, Environmental sustainability, Green care, Quality improvement

\section{INTRODUCTION}

Until recently, health and health care were relatively low on the international climate agenda. This is gradually changing, which makes sense in view of recent worldwide societal and political unrest regarding climate change, in particular with regard to the Covid-19 pandemic that is allegedly linked to it. $^{[1]}$ There is mounting evidence for the negative effects of climate change and pollution on a range of health outcomes.
Global warming is directly and indirectly associated with increases in cardiovascular and pulmonary disease, mental disease, cancer, diabetes and zoonoses, before even taking account of the consequences of water shortages and food insecurity. ${ }^{[2]}$ Over recent years, the Lancet Countdown initiative and other authoritative sources have warned about the negative health impact if temperatures continue to rise and greenhouse gas emissions are not reduced. ${ }^{[3]}$ In fact, the

*Correspondence: Hans C. Ossebaard; Email: hossebaard@zinl.nl; Address: National Health Care Institute, P.O. Box 320, 1110 AH, Diemen, The Netherlands. 
health gains of the last fifty years are under severe threat from climate change and a global effort therefore needs to lead to greater sustainability and less environmental impact from human activities. ${ }^{[4]}$ Many other scientific reports have confirmed these conclusions despite ongoing disinformation campaigns. Health scientists today explicitly refer to "a health emergency." ${ }^{[5]}$ It is also recognized that the global health care industry plays a substantial role in climate change through greenhouse gas emissions, waste and pharmaceutical pollution, ${ }^{[6]}$ to a much greater extent (obviously) in high-resource countries than in low-resource countries.

In the Netherlands too, sustainability and climate change have become key policy issues. The national government has signed the international Paris Agreement, part of the UN Climate convention, ${ }^{[7]}$ and other international treaties. Besides that, the Dutch coalition government is aiming to reduce $\mathrm{CO}_{2}$ emissions by $49 \%$ by $2030^{[8,9]}$ and a national strategy has been designed for implementing climate mitigation and adaptation measures in collaboration with all the stakeholders involved. ${ }^{[10,11]}$

The Dutch health care sector has only recently started actively and visibly participating in this development. The carbon footprint of the Dutch health care sector is estimated to be approximately $7 \%$ of the total national net emissions, ${ }^{[12]}$ which is high compared with other countries. ${ }^{[13]}$ To counter this, a range of policy measures and professional initiatives are either being prepared or are in effect today. ${ }^{[14]}$ The Ministry of Health is aiming to accelerate this through the "Sustainable care for a healthy future" Green Deal. Since 2015, this novel collaborative policy instrument has been helping stakeholders from government, care providers, education and the commercial sector to improve sustainability in health care. Over 200 parties have signed a covenant and pledged to take appropriate action. Among them is the Dutch National Health Care Institute, an independent government body with statutory tasks regarding the health care insurance package and health care quality. ${ }^{[15]}$ To underline its commitment, this institute commissioned an explorative study to examine the options for embedding environmental sustainability in its mission. ${ }^{[16]}$

Sustainability is a somewhat indefinite concept, but the present paper defines it as "meeting the resource and service needs of current and future generations without compromising the health of the ecosystems that provide them."[17] As one of the main tasks of the National Health Care Institute is to encourage improvements in health care quality, ${ }^{[15]}$ this study focuses on how quality correlates with environmental sustainability in scientific literature, with quality of care being defined as "the extent to which health care services provided to individuals and the patient population improve desired health outcomes. To achieve this, health care must be safe, effective, in time, efficient, equitable and peoplecentred." ${ }^{218]}$ We believe that the conceptualization of environmental impact of health care as an inherent aspect of quality in care would be meaningful for theoretical and practical reasons. Conversely, conceptualizing quality of care as including green measures in several aspects of care could influence the way we think about health care quality improvement. Climate change is the greatest challenge of our times. Societies, science and policy as well as our health care systems must respond with effective measures for adapting and for mitigating its effects, without being disrupted themselves. In view of the present health emergency situation we must reconsider our health care delivery systems. Hence it is important to understand how the basic concepts of quality and sustainability are related, and what this implies for health care practice.

As early as 2001, researchers were calling for sustainability to be included as an element of health care quality ${ }^{[19]}$ and this has been elaborated upon in later studies by several authors. Recently, the British Royal College of Physicians incorporated sustainability as a new dimension of health care quality, ${ }^{[20]}$ inspired by what what is considered to be "one of the defining issues of our time" and "one of the world's most urgent health threats." ${ }^{[21]}$ The present study aims to assess how environmental sustainability (hereinafter simply referred to as "sustainability") correlates with quality of care in academic literature and to identify possible conflicts between the two concepts. The findings are followed by a discussion of the possible implications for science, policy and health care.

\section{METHOD}

To examine the conceptual relationship between "sustainability" and "quality of care," we carried out a scoping review. This type of literature research is useful for summarizing academic literature, especially when the topic of interest has not been comprehensively reviewed before, ${ }^{[22,23]}$ as is the case here. The review is aimed at finding associations and conflicts between environmental sustainability and commonly-used dimensions of health care quality such as safety, effectiveness, timeliness, efficiency, equity and patient-centredness. ${ }^{[18]}$ A distinction is made between collecting data for associations and collecting data for conflicts between sustainability and quality of care. The way the search process is conducted is similar, but different search strings were applied. 


\subsection{Data sources}

Data was collected via Scopus, PubMed and Google Scholar in May 2019. Before collecting the data, a cyclic iterative search of the three databases was done in order to identify relevant search terms and to determine inclusion and exclu- sion criteria. A total of 57 relevant search terms were found, which were then structured in a semantic network (see Figure $1)$.

The inclusion and exclusion criteria are outlined in Table 1.

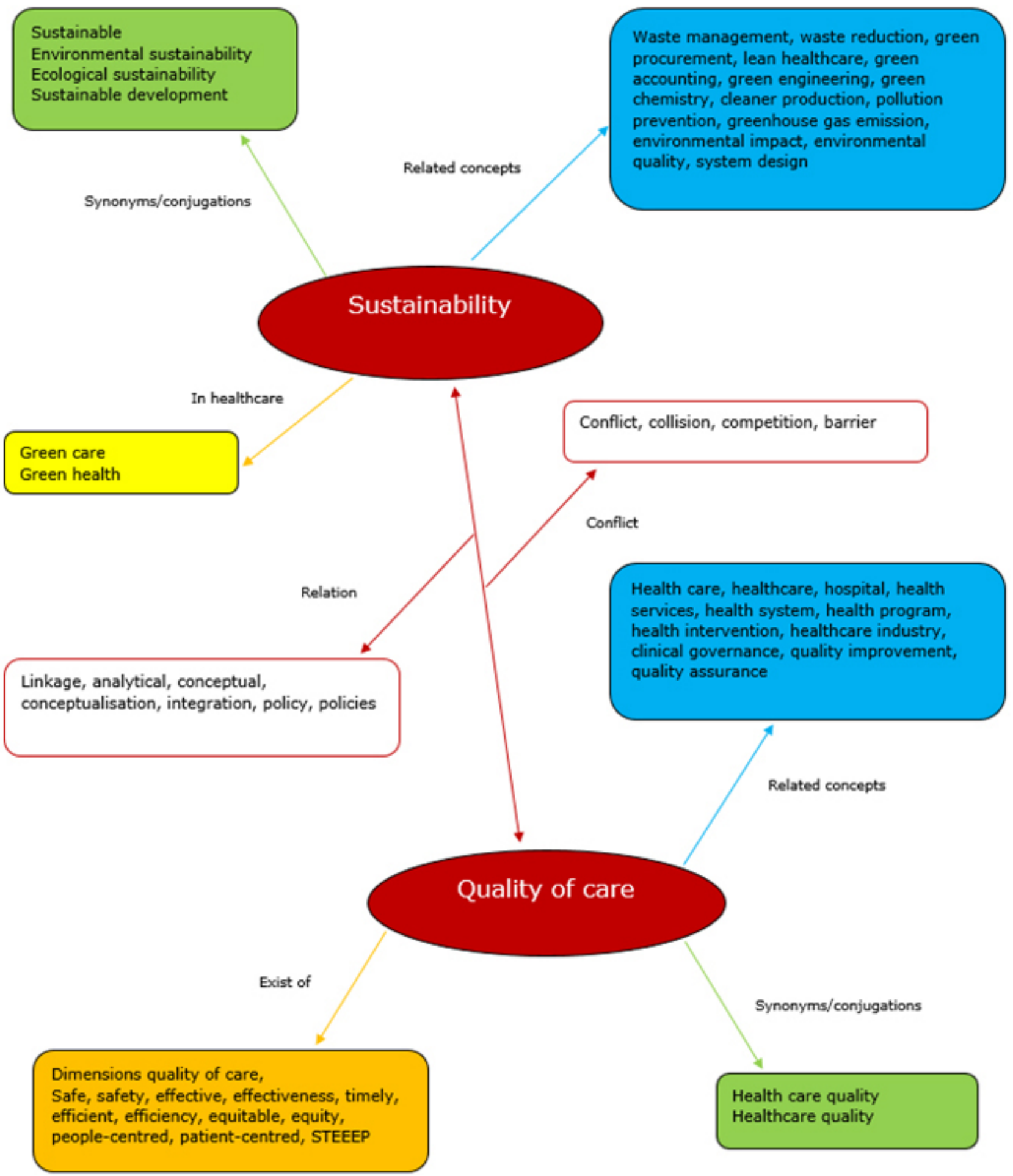

Figure 1. Semantic network of search terms 
Table 1. Inclusion and exclusion criteria

\begin{tabular}{cl}
\hline Inclusion criteria & Exclusion criteria \\
\hline • Year of Publication between 2010-2019 & • Interpretation of sustainability as “endurance,” "continuity” or “maintenance” \\
• Article contains keywords related to both & • Sustainability as practical application (e.g., sustainability programmes) \\
sustainability and healthcare & $\bullet$ Interpretation of sustainability as a financial or economical parameter \\
\hline
\end{tabular}

\subsection{Study selection}

Search strings were created withvarious search terms and entered into PubMed and Scopus. Google Scholar was merely consulted (the first 100 hits) as an addition to the outcomes of the two academic databases, as searching this database can only be done less systematically because of the way it is structured. Articles were first screened for relevance by reading the titles and abstracts and then by examining the full contents.

For the first part, about associations between sustainability and quality of care, a total of 11 search strings were defined and entered into PubMed and Scopus based on the search terms (see Appendix: Supplementary data). As a result, 1,488 studies were identified after eliminating duplicates (see Figure 2). After screening the title and abstract, 8 studies remained. An additional 13 articles were included through Google Scholar. Of these 21 articles, 11 were retained for analysis after reading the full content.

For the second part, about conflicts between sustainability and quality of care, 4 search strings were defined using the search terms. A total of 45 studies were identified of which 19 were duplicates, so a total of 26 studies remained from Scopus and PubMed. One more study was added through Google Scholar and another was found during data collection for the first part about associations between sustainability and quality of care. After screening the titles and abstracts, 6 studies remained; after scanning the full content of these studies, 3 studies failed the inclusion criteria and were eliminated. In the end, 3 studies remained for analysis.

\subsection{Data extraction}

A data extraction form was developed for this study, in which the most relevant information from each study was summarized. The form consists of two categories; one for associations (interpretation and definition of sustainability, and quality of care or dimensions thereof), the other concerning potential conflicts (tension between sustainability and quality of care or dimensions thereof). Differences, similarities and patterns were identified between the various studies and the most important findings were summarized accordingly.

\section{RESULTS}

Although both "sustainability" and "health care" were addressed in the selected studies, almost none specifically refer to quality of care. Most authors report their perspectives on sustainability, for which a balance between economic, social and environmental domains - the "three pillars of sustainability" - is elaborated upon most often. Some argue that such a balance is needed to achieve a future-proof health care system. ${ }^{[24]}$ Other studies consider sustainability to be an "overarching concept" that is important in various processes of the health care sector, e.g., production, treatments, travel movements. The health care industry is a complex and adaptive sector that needs to examine new practices and technologies and carries out activities to improve costeffectiveness, safety and quality. Therefore, sustainability in health care is related to a range of quality improvement pathways. ${ }^{[25]}$ This is in line with Marimuthu and Paulose, ${ }^{[26]}$ who report that a multi-directional focus is needed for health care organizations to be sustainable.

Other studies do not recognize a relationship between sustainability and quality of care. It appears that health care professionals and nurses do not seem to consider sustainability a number one priority as they habitually rely on a biomedical model of health with no explicit role for the environment. ${ }^{[27,28]}$ Besides that, even if they want to implement sustainability in their workplace, they report workplace culture and professional identities as barriers against its implementation. ${ }^{[27]}$

Eleven studies provide indications of a relationship between sustainability and some dimensions of quality. The dimensions derived from these studies are efficiency, patientcentredness and safety. In contrast, only three studies were found that are sceptical about a relationship between sustainability and efficiency or effectiveness. Results are discussed in more detail below. For the other two commonly used dimensions, equity and timeliness, we found neither indications for a positive nor a negative relationship with sustainability.

\subsection{Efficiency}

Efficiency is about delivering health care in a way that maximizes resource use and avoids waste. 


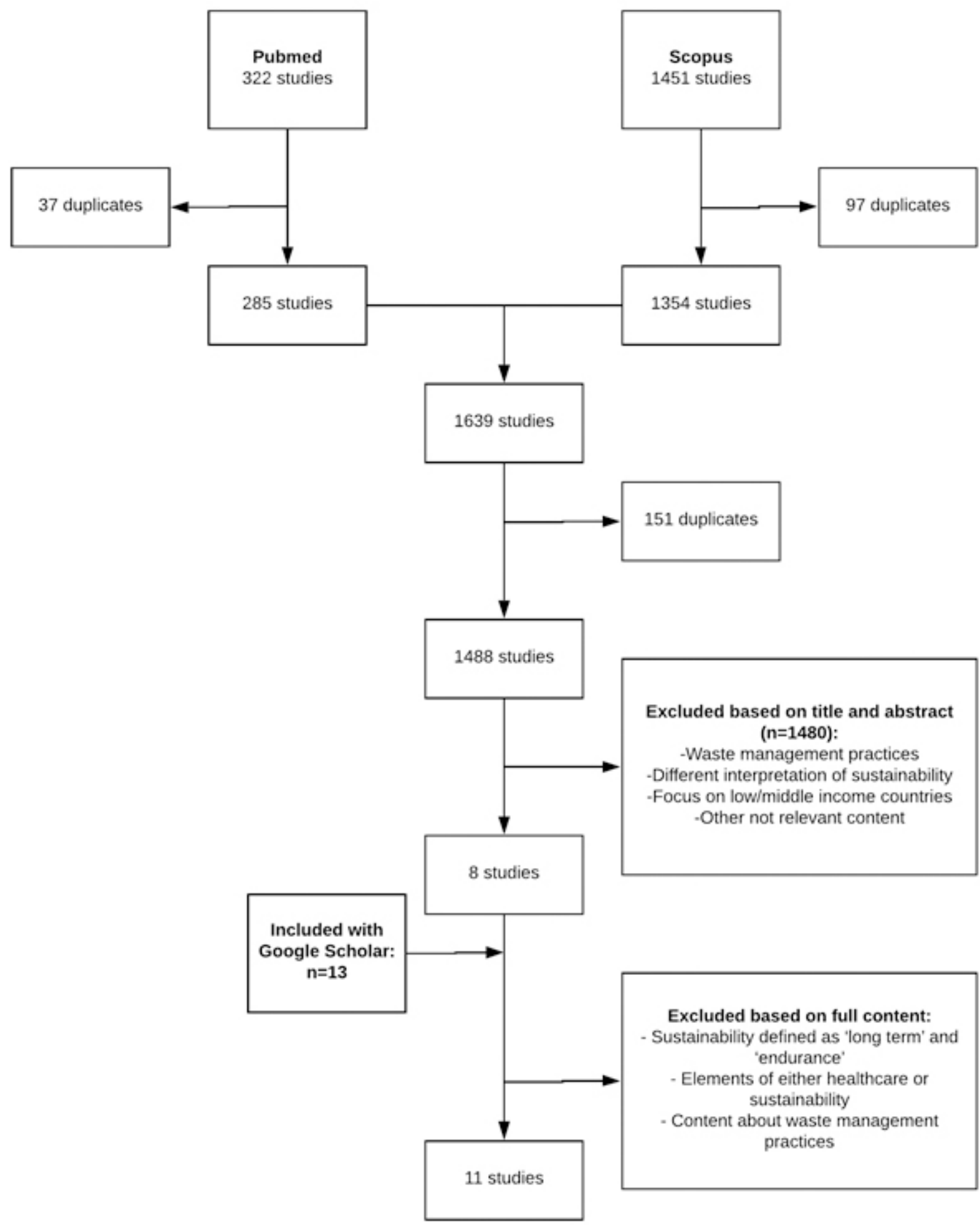

Figure 2. Flowchart of data collection regarding the associations between sustainability and quality of care

\subsubsection{Associations with sustainability}

Most studies that connect sustainability with a dimension of quality of care mention efficiency, which is about maximizing resources and avoiding waste. ${ }^{[29]}$ The $\mathrm{WHO}^{[29]}$ mentioned the fact that efficiency can influence environmental impact, as an inefficient health system typically generates environmental and financial waste. Sustainable health care facilities ensure that energy is saved, natural resources are preserved and waste is segregated. ${ }^{[30]}$ A concept closely related to efficiency covers data-driven, statistical quality 
improvement strategies such as Lean or Six Sigma as applied in health care. These methods are all about eliminating waste in the form of artificial variety in order to achieve high-value care and safety for patients. ${ }^{[24,31,32]}$ This is in line with the commonly used IOM definition of efficiency, "Delivering health care in a way that maximizes resource use and avoids waste.’[18]

\subsubsection{Conflicts with sustainability}

While sustainability is often associated with efficiency or effectiveness, there are some distinctions between the three concepts. ${ }^{[33]}$ Sustainability is seen as a holistic concept that refers to a large space with many stakeholders involved in an ecosystem and with a long time-span. ${ }^{[33]}$ Efficiency is generally used to quantify measurable aspects of performance such as speed or time. As the efficiency concept is more tangible than sustainability, it can be quantified in a limited timeframe. The different timeframes make it difficult to further blend the two concepts.

\subsection{Patient-centredness}

Patient-centredness is about providing care that takes into account the preferences and aspirations of individual service users and the culture of their community. ${ }^{[18]}$

\section{Associations with sustainability}

Some studies indicate a correlation between sustainability and patient-centredness. Patients are important stakeholders in achieving sustainable health care as they are the focus of care and they have expectations and experiences related to costs, wellbeing and accessibility. ${ }^{[25,34]}$ Faezipour and Ferreira point out that patient satisfaction is a social dimension of sustainability in health care. ${ }^{[25]}$ Instead of considering patients as determinants of sustainable health care, a WHO report ${ }^{[35]}$ correlates an increase in patient knowledge and participation with a decrease in environmental impact. It is hypothesized that empowered patients could manage their own health more efficiently, communicate their needs and preferences to professionals better and support the health of community members by making less use of health system resources. ${ }^{[35]}$ More research is needed to support this interesting supposition.

\subsection{Safety}

Safety is about delivering health care that minimizes risks and harm to service users, including avoiding preventable injuries and reducing medical errors. ${ }^{[18]}$

\section{Associations with sustainability}

A few studies recognize a correlation between safety and sustainability. Environmental sustainability could improve health and safety of both health care providers and pa- tients. ${ }^{[36]}$ Health care providers perform tasks that are sometimes hazardous and they may suffer minor or major injuries from using medical devices, musculoskeletal injuries as a result of falls, or toxic exposure to harmful chemicals. Although the relationship is not very well understood yet, promoting sustainable activities could protect the health of patients and their health care providers. ${ }^{[36]}$ Some examples mentioned are (a) the use of "green" cleaners that positively affect illness and asthma, (b) waste management that reduces employee exposure to infectious diseases and (c) reducing the number of cars around a hospital in order to improve respiratory health outcomes. This is in line with a paper that advocates a high level of safety and minimum risk for all stakeholders to achieve sustainable health care. ${ }^{[30]}$

\subsection{Effectiveness}

Effectiveness is about providing services, based on scientific knowledge and evidence-based guidelines, that meet the need of the patient. ${ }^{[18]}$

\section{Conflicts with effectiveness}

Effectiveness is a composite concept that includes the usefulness and relevance of the outcomes of care, e.g., doing the right thing at the right time and the right place. As mentioned in 3.1.2, sustainability is defined at a different level and implies a longer time-span. ${ }^{[33]}$ The differences in timeframe between sustainability on the one hand and effectiveness on the other overlap with Dunphy, ${ }^{[27]}$ who notes that health care often focuses on short-term outcomes that can be assessed relatively easily at an individual level, such as patient satisfaction or economic outcomes. Environmental impact and sustainability refer to the longer term, at the population level, which may be perceived as less relevant by health care professionals. ${ }^{[27]}$

\section{Discussion}

This study aims to explore the correlation between environmental sustainability and quality of care by studying possible associations and conflicts between the two concepts. The main outcome is that only a few studies were identified that actually report on both concepts, and their relationship remains quite unclear. This could be explained by the ambiguity of the concept of sustainability, which lacks an intersubjective definition in health care. The studies included used various interpretations of sustainability, as also found by White ${ }^{[37]}$ who identified 100 different definitions of sustainability. Moreover, "sustainability" and "sustainable development" are often used interchangeably, even in peerreviewed articles, which suggests that students, researchers, professors and journal editors are confused about definitions of sustainability. ${ }^{[38]}$ It is difficult to study a relationship 
without understanding its component elements.

Nevertheless, some studies do address the relationship between sustainability and quality of care, or at least report some ideas about it. One major finding is that these studies show almost no consensus on what such a relationship could look like. For example, efficiency is mentioned as both a connecting and a conflicting dimension. On the one hand, efficiency is about maximizing the use of resources and avoiding waste, which is perceived as sustainable. On the other hand, efficiency and sustainability are thought to operate at different levels, namely short-term versus longterm outcomes. Patient satisfaction is generally associated with the levels of politeness, friendliness and communication demonstrated by nurses during treatment. ${ }^{[39]}$ It may be associated with sustainability as well if patients are acting as ambassadors for promoting sustainable health care, but there are different opinions about the exact role patients could take. Some believe it is enough to have a clear picture of patients' experiences, while others argue for a more active role for the patients by empowering them to decrease environmental impact.

Safety seems to have a synergistic relationship with sustainability, as several authors argued that greater sustainability could lead to a safer environment for both patients and health care workers. However, it is unclear whether the reverse relationship is also valid, i.e., that a safer environment leads to greater sustainability in health care. A paper by Mortimer et al. ${ }^{[20]}$ stands out in terms of the sophistication and elaboration of sustainability as an important value in health care. While a scoping review was chosen as the most appropriate method for the present explorative study, it does not allow for exhaustive systematic searching; we examined only three databases in a short timeframe. This key paper ${ }^{[20]}$ initially escaped our search. It appeared to be the only in-depth contribution that seeks to integrate environmental impact into a new framework to assess health care quality. The triple bottom line of this framework designates a new value, as it includes not only the social impact and economic cost of health care but also its environmental impact. This innovative attempt to advance eco-friendliness as a quality value in care is gradually receiving more attention in the academic world and in the international quality improvement movement. ${ }^{[40]}$

In view of the disastrous consequences of climate change and pollution on health, the health care industry faces the challenge of reducing its ecological footprint. The public health response to the Covid-19 crisis has accentuated this. Increasing sustainability at all levels of the organization is the approach to achieving this. Environmental impact could well be viewed as a dimension of health care quality, quality improvement projects could therefore very well include sustainability. In this respect, the results of the review reveal a blind spot in our knowledge, as only a few studies report about this relationship and there is no consensus among the ones that did. Given the fact that health and health care have only recently been prioritized on the climate change agenda, this is no surprise. Research about the relationship between sustainability and health care is still at an early stage; the results of this review clearly indicate this. If we are to improve our understanding of environmental sustainability as an achievable objective in quality improvement, we need more research into its applicability and its implication at the individual and organizational levels. Numerous practical examples show alternatives for conventional treatments that have far less environmental impact while retaining the same levels of effectiveness and safety for the patient. There is a question of the extent to which sustainability is suitable for implementing in one or more of the dimensions of health care quality, or for inclusion as an additional dimension, as suggested by Mortimer et al. ${ }^{[20]}$ In the meantime, some promising initiatives for implementing "green care" have been taken by health care parties, which is a positive sign that underscores the increasing sense of urgency for advancing sustainability in health care.

\section{ACKNOWLEDGEMENTS}

The authors would like to thank the National Health Care Institute for the opportunity to carry out this study and to present, report and discuss its outcomes.

\section{CONFLiCTS OF INTEREST Disclosure}

The authors declare no conflicts of interest.

\section{REFERENCES}

[1] Hughes JM, Wilson ME, Pike BL, et al. The origin and prevention of pandemics. Clinical Infectious Diseases. 2010; 50: 1636-40. PMid:20450416. https://doi.org/10.1086/652860

[2] Watts N, Adger WN, Agnolucci P, et al. Health and climate change policy responses to protect public health. Lancet. 2015; 386: 1861914. https://doi.org/10.1016/S0140-6736(15) 60854-6
[3] Watts N, Amann M, Arnell N, et al. The 2018 report of the Lancet Countdown on health and climate change: shaping the health of nations for centuries to come. Lancet. 2018; 392: 2479-514. https://doi .org/10.1016/S0140-6736 (18) 32594-7

[4] Watts N, Amann M, Arnell N, et al. The 2019 report of The Lancet Countdown on health and climate change: ensuring that the health of a child born today is not defined by a changing climate. The 
Lancet. 2019; 394: 1836-78. https://doi.org/10.1016/S014 0-6736 (19) 32596-6

[5] Haines A, Ebi K. The imperative for climate action to protect health. New England Journal of Medicine. 2019; 380: 263-73. PMid:30650330. https://doi.org/10.1056/NEJMra1807873

[6] Bosurgi R. Climate crisis: healthcare is a major contributor, global report finds. BMJ. 2019; 366: 55-60. PMid:31519554. https: //doi.org/10.1136/bmj.15560

[7] United Nations Framework Convention on Climate Change. The Paris Agreement. 12 October 2019. Available from: https://unfccc.int/process-and-meetings/the-paris -agreement/the-paris-agreement

[8] Koelemeijer R, Daniëls B, Boot P, et al. Analysis of Coalition Agreement Rutte-III: Effects on climate and energy [Analyse reageerakkoord Rutte-III: effecten op klimaat en energie]. Den Haag: PBL Netherlands Environmental Assessment Agency; 2017.

[9] Dutch National Government. Climate Policy. 12 October 2019 Available from: https://www.government.nl/topics/climate-c hange/climate-policy

[10] Knowledge Portal Spatial Adaptation. National Climate Adaptation Strategy. 21 October 2019. Available from: https://ruimtelijk eadaptatie.nl/english/policy-programmes/nas/

[11] Traub J. There's only one way for democracies to save the planet. Foreign Policy. Dec 21, 2019.

[12] Gupta Strategists. Transition to a sustainable health care sector. 12 September 2019. Available from: https://gupta-strategists.nl/en/research/een-stuur -voor-de-transitie-naar-duurzame-gezondheidszorg

[13] Harm HCW. Health Care's Climate Footprint: how the health sector contributes to the global climate crisis and opportunities for action. 2019.

[14] Ossebaard HC. Online Column Mobile Doctors: Green Care. 12 October 2019. Available from: https://www.mobiledoctors.nl/2 018/06/column-groene-zorg/

[15] National Health Care Institute. Tasks of the National Health Care Institute. 21 October 2019. Available from: https://english.zorginstituutnederland.nl/about-us/ tasks-of-the-national-health-care-institute

[16] Veltman LMS. Real Deal: Exploring Green Policy Options for the Dutch National Health Care Institute: MSc Internship Wageningen University \& Research / Dutch National Health Care Institute; 2019.

[17] Morelli J. Environmental sustainability: A definition for environmental professionals. Journal of Environmental Sustainability. 2011; 1: 2. https://doi.org/10.14448/jes.01.0002

[18] Institute of Medicine. Crossing the Quality Chasm: A New Health System for the 21st Century. Washington DC: National Academy Press; 2001.

[19] Kaiser B, Eagan PD, Shaner H. Solutions to health care waste: lifecycle thinking and green purchasing. Environmental Health Perspectives. 2001; 109: 205-7. PMid:11333178. https://doi.org/10.1 289/ehp. 01109205

[20] Mortimer F, Isherwood J, Wilkinson A, et al. Sustainability in quality improvement: redefining value. Future Hospital Journal. 2018; 5: 88-93. PMid:31098540. https://doi.org/10.7861/futureho sp. 5-2-88

[21] World Health Organisation (WHO). 21 October 2019. Available from: https://www.who.int/news-room/detail/11-09-2 019-who-director-general-urges-world-leaders-to- $p$ rotect-health-from-climate-chang

[22] Galway LP, Beery T, Jones-Casey K, et al. Mapping the solastalgia literature: A scoping review study. International Journal of Environ- mental Research and Public Health. 2019; 16: 2662. PMid:31349659. https://doi.org/10.3390/ijerph16152662

[23] Peters MD, Godfrey CM, Khalil H, et al. Guidance for conducting systematic scoping reviews. International Journal of Evidence-based Healthcare. 2015; 13: 141-6. PMid:26134548. https://doi.org/ $10.1097 /$ XEB. 0000000000000050

[24] Boone T. Creating a Culture of Sustainability: leadership, coordination and performance measurement decisions in healthcare. 12 September 2019. Available from: https: //www. chausa.org/docs/default-source/environment/ creating-a-culture-o-sustainability.pdf?sfvrs

[25] Faezipour M, Ferreira S. A system dynamics perspective of patient satisfaction in healthcare. Procedia Computer Science. 2013; 16 148-56. https://doi.org/10.1016/j.procs . 2013.01.016

[26] Marimuthu M, Paulose H. Emergence of sustainability based approaches in healthcare: Expanding research and practice. ProcediaSocial and Behavioral Sciences. 2016; 224: 554-61. https://doi. org $/ 10.1016 / j$. sbspro. 2016.05.437

[27] Dunphy JL. Healthcare professionals' perspectives on environmental sustainability. Nursing Ethics. 2014; 21: 414-25. PMid:24106259. https://doi.org/10.1177/0969733013502802

[28] Griggs C, Fernandez A, Callanan M. Nursing and the barriers to sustainable health care: a literature review. British Journal of Nursing 2017; 26: 1230-7. PMid:29240479. https://doi.org/10.12968 /bjon.2017.26.22.1230

[29] World Health Organisation. What is quality of care and why is it important. 27 September 2019. Available from: https://www.who.int/maternal_child_adolescent/topi cs/quality-of-care/definition/en/

[30] Waste Management. Healthcare and the triple bottom line. 12 October 2019. Available from: https://www.wm.com/us

[31] Hwang P, Hwang D, Hong P. Lean practices for quality results: a case illustration. International Journal of Health Care Quality Assurance. 2014; 27: 729-41. PMid:25417378. https://doi.org/10.1108/ I JHCQA-03-2014-0024

[32] Singh P. Lean in healthcare organization: an opportunity for environmental sustainability. Benchmarking: An International Journal. 2019; 26: 205-20. https://doi .org/10.1108/BIJ-04-2018-0104

[33] Nayak AK. Efficiency, effectiveness and sustainability: the basis of competition and cooperation. XIMB Sustainability Seminar Series, Working Paper. 2011; 3.

[34] Saviano M, Bassano C, Calabrese M. A VSA-SS approach to healthcare service systems the triple target of efficiency, effectiveness and sustainability. Service Science. 2010; 2: 41-61. https: //doi.org/10.1287/serv.2.1_2.41

[35] World Health Organisation. Towards environmentally sustainable health systems in Europe: a review of the evidence. 27 September 2019. Available from: http://www . euro. who.int/en/health-topics/enviro nment-and-health/Climate-change/publications/2016/ towards-environmentally-sustainable-health-systems -in-europe.-a-review-of-the-evidence-2016

[36] Kaplan SB, Forst L. Linking Environmental Sustainability, Health, and Safety Data in Health Care: A Research Roadmap. NEW SOLUTIONS: A Journal of Environmental and Occupational Health Policy. 2017; 27: 189-209. PMid:28541159. https://doi.org/10.117 7/1048291117710784

[37] White MA. Sustainability: I know it when I see it. Ecological Economics. 2013; 86: 213-7. https ://doi .org/10.1016/j.ecolec on. 2012.12 .020 
[38] Salas-Zapata WA, Ortiz-Muñoz SM. Analysis of meanings of the concept of sustainability. Sustainable Development. 2019; 27: 153-61. https://doi.org/10.1002/sd.1885

[39] Rademakers J, Delnoij D, de Boer D. Structure, process or outcome: which contributes most to patients' overall assessment of healthcare quality? BMJ Quality \& Safety. 2011; 20: 326-31. PMid:21339310. https://doi.org/10.1136/bmjqs.2010.042358

[40] Ossebaard HC, Lachman P. Climate change, environmental sustainability and health care quality. International Journal of Quality in Health Care. 2020; 32: 1-3. https://doi.org/10.1093/intqhc /mzaa036 